\title{
Silk fibroin hydrolysate inhibits osteoclastogenesis and induces apoptosis of osteoclasts derived from RAW 264.7 cells
}

\author{
JEON-WOO CHON ${ }^{1}$, HYERYEON KIM ${ }^{2}$, HA-NA JEON ${ }^{2}$, KYUNGHO PARK ${ }^{2}$, KWANG-GILL LEE ${ }^{1}$, \\ JOO-HONG YEO $^{1}$, HAEYONG KWEON ${ }^{1}$, HEUI-SAM LEE ${ }^{1}$, YOU-YOUNG JO ${ }^{1}$ and YOO KYOUNG PARK ${ }^{2}$ \\ ${ }^{1}$ Sericultural and Apicultural Materials Division, National Academy of Agricultural Science, RDA, Suwon 441-100; \\ ${ }^{2}$ Department of Medical Nutrition, Graduate School of East-West Medical Science, \\ Kyung Hee University, Yong-in 446-701, Republic of Korea
}

Received November 19, 2011; Accepted January 2, 2012

DOI: $10.3892 /$ ijmm.2012.1120

\begin{abstract}
Bone disease can be associated with bone resorption by osteoclasts, and interest in the development of antiresorptive agents has recently increased. The hydrolysate of silk fibroin has been studied with respect to such biomedical applications. In a previous study, silk fibroin showed indirect inhibitory effects on the differentiation of osteoclasts. To further evaluate the effect of a hydrolysate of silk fibroin on osteoclasts, we investigated the direct effects of the silk fibroin hydrolysate on osteoclastogenesis and apoptosis of osteoclasts induced by receptor activation of nuclear factor $\kappa \mathrm{B}$ ligand (RANKL). The silk fibroin hydrolysate inhibited RANKL-induced formation of tartrate-resistant acid phosphatase (TRAP) in RAW 264.7 cells. The inhibitory effect of the silk fibroin hydrolysate resulted in the decreased expression of osteoclast marker genes, such as matrix metalloproteinase-9 (MMP-9), cathepsin-K and calcitonin receptor (CTR). In addition, the silk fibroin hydrolysate blocked the signaling pathways of mitogen-activated protein kinase (MAPK) and nuclear factor- $\kappa \mathrm{B}(\mathrm{NF}-\kappa \mathrm{B})$ and expression of transcription factors, such as nuclear factor of activated $\mathrm{T}$ cells $\mathrm{c} 1$ (NFATc1) and NF- $\mathrm{KB}$. Finally, the silk fibroin hydrolysate induced apoptosis signaling cascades. Taken together, the present results indicate that silk fibroin hydrolysate has antiresorptive activity by both inhibiting osteoclastogenesis and inducing osteoclast apoptosis.
\end{abstract}

\section{Introduction}

Bone is continuously remodeled through bone formation by osteoblasts and bone resorption by osteoclasts. A balance of bone remodeling is important for maintaining skeletal strength

Correspondence to: Dr Yoo Kyoung Park, Department of Medical Nutrition, Graduate School of East-West Medical Science, Kyung Hee University, 1 Seocheon-dong Giheung-gu, Gyeonggi, Yong-in 446-701, Republic of Korea

E-mail: mednutr@nate.com

Key words: osteoclastogenesis, apoptosis, receptor activation of nuclear factor $\kappa \mathrm{B}$ ligand, osteoclast, hydrolysate silk fibroin and density (1). However, an imbalance in bone remodeling caused by excessive osteoclastic bone resorption leads to bone destructive diseases, such as osteoporosis (2).

Osteoclasts originate from hematopoietic precursor cells of the monocyte/macrophage series and differentiate into bone-resorbing multinucleated cells by fusion of mononuclear cells. The osteoclastic process consists of multiple steps, including osteoclastogenesis, activation, and apoptosis (3). Osteoclasts differentiate from preosteoclasts in the presence of receptor activator of nuclear factor $-\kappa B$ ligand (RANKL). Binding of RANKL to its receptor activates tumor necrosis factor receptor-associated factor 6 (TRAF6), which is induced through the activation of nuclear factor $-\kappa \mathrm{B}(\mathrm{NF}-\kappa \mathrm{B})$ via the inhibitor of NF- $\kappa \mathrm{B}(\mathrm{I} \kappa \mathrm{B})$ kinase and mitogen-activated protein kinases (MAPK) (4). In addition, activated extracellular signal-regulated kinase (ERK) can phosphorylate c-Fos, which subsequently phosphorylates the nuclear factor of activated $\mathrm{T}$ cells (NFATc) transcription factor (5). Therefore, RANKL induces transcription factors required for the regulation of genes involved in osteoclast differentiation. These osteoclasts are characterized by expression of the markers tartrate-resistant acid phosphatase (TRAP), matrix metalloproteinase-9 (MMP-9), cathepsin-K, and calcitonin receptor (CTR) (6).

Osteoclasts subsequently undergo apoptosis, which is programmed cell death characterized by cell chromatin condensation, cell shrinkage, and nuclear fragmentation (7). Osteoclast apoptosis is related to bone remodeling and the treatment of bone diseases (8). Osteoclasts possess general apoptotic mechanisms. The Fas receptor has been identified in mouse and human osteoclasts and in RAW 264.7 cells, and functions through a Fas signaling pathway (9) that is regulated by the Bcl-2 protein, which in turn controls the release of cytochrome $\mathrm{c}$ from mitochondria. Caspase proteins are activated via cytochrome $\mathrm{c}$, and apoptosis is typically defined by activated caspase-3 (10). The relative rate of pro-apoptotic and anti-apoptotic events determines susceptibility to death signals.

In recent years natural compounds that inhibit bone resorption have been studied for their ability to reduce the rate of osteoclast differentiation or the number of activated osteoclasts, or to induce osteoclast apoptosis. Among these, agents that inhibit osteoclast differentiation have been proposed to prevent and treat osteoporosis (11). Prior studies associated 
with inhibition of osteoclastogenesis can be divided into two groups. It was reported that osteoclasts interact closely with osteoblasts. RANKL, a member of the TNF family, is a significant factor in osteoclast differentiation. RANKL is expressed by osteoblasts/stromal cells, and its expression is increased by factors including $1,25(\mathrm{OH})_{2} \mathrm{D}_{3}$, prostaglandin $\mathrm{E}_{2}\left(\mathrm{PGE}_{2}\right)$, parathyroid hormone (PTH), and interleukin-11 (IL-11). Osteoprotegein (OPG) is a decoy receptor for RANKL that negatively regulates bone resorption by binding to RANKL. Thus, in many environments, osteoclastogenesis is regulated indirectly by osteoblasts/stromal cells (12). Therefore, natural extracts such as soybean and Scutellaria radix used in the treatment of osteoblasts have indirect inhibitory effects on osteoclastogenesis. An alternative method for demonstrating direct effects on the inhibition of osteoclastogenesis is through blockage of signaling pathways induced by RANKL in RAW 264.7 cells $(13,14)$.

Silk is a well known fibrous protein produced by the silkworm that has been used traditionally as textile fibers and surgical sutures for humans (15). It is composed of two kinds of proteins, a filament core protein (fibroin) and a gum-like coating protein (sericin) that surrounds the fibroin fibers to cement them together. Silk fibroin consists of heavy and light chain polypeptides of $\sim 390$ and $\sim 25 \mathrm{kDa}$, respectively, linked by a disulfide bond at the $\mathrm{C}$-termini of the two subunits through hydrophobic interactions. The hydrophobic blocks tend to form $\beta$-structures or crystals through hydrogen bonding and hydrophobic interactions, forming the basis for the tensile strength of silk fibroin (16). Hydrolysates of silk fibroin are water-soluble peptides that have been investigated as food and dietary supplements. Hydrolysate silk fibroin contains 18 kinds of amino acids including Gly (45.9\%), Ala (30.3\%), Ser $(12.1 \%)$, Tyr $(5.3 \%)$ and Val $(1.8 \%)(17,18)$. Numerous studies have documented a range of effects of hydrolysate silk fibroin, including moisturizing (19), wrinkle improvement, inhibition of tyrosinase (20), and apoptosis (21), and biomedical applications such as a matrix for mammalian cell culture and enzyme immobilization (22), a scaffold for bone substitution (23), a wound dressing (24), and a drug delivery carrier (25). Silk fibroin hydrolysate has also been used in biomedical materials, such as bone, cartilage, and ligaments (26). In a previous study, we demonstrated the indirect effect of silk fibroin on the inhibition of osteoclastogenesis in RAW 264.7 cells (27). These results led us to investigate the direct effect of hydrolysate silk fibroin on osteoclastogenesis.

Therefore, in this study we investigated the effect of hydrolysate silk fibroin on signaling pathways involving ERK1/2 and $\mathrm{NF}-\kappa \mathrm{B}$ and the induction of apoptosis. Mouse RAW 264.7 cells were grown to fully differentiate into osteoclasts and expression levels of genes such as MMP-9, TRAP, cathepsin-K and CTR, which are well known markers for osteoclasts, were also measured.

\section{Materials and methods}

Preparation of extract from silk fibroin. Raw silk (Bombyx mori $L$.) cocoons reared on a farm affiliated with the Rural Development Administration of Korea were used as the raw materials. The raw materials were degummed twice with $0.5 \%$ on the weight of fiber (OWF) Marseilles soap and a $0.3 \%$ OWF sodium carbonate solution at $100^{\circ} \mathrm{C}$ for $1 \mathrm{~h}$ and then washed with distilled water. Degummed silk fibroin fibers were dissolved in a mixed solution of $\mathrm{CaCl}_{2}, \mathrm{H}_{2} \mathrm{O}$, and ethanol at $95^{\circ} \mathrm{C}$ for $5 \mathrm{~h}$. This calcium chloride/silk fibroin mixed solution was filtered twice through a Miracloth (Calbiochem, San Diego, CA, USA) quick filter. For the desalting of the calcium chloride/silk fibroin mixed solution, gel filtration column chromatography was performed on a GradiFrac system (Amersham Parmacia Biotech, Tokyo, Japan) equipped with a UV-1 detector operating at $210 \mathrm{~nm}$. A commercially available and prepacked Sephadex G-25 column (800x40 mm i.d. Amersham Parmacia Biotech) was used. Distilled water was used as the elution solvent at a flow rate of $25 \mathrm{ml} / \mathrm{min}$; the sample injection volume was $200 \mathrm{ml}$, and the fraction volume was $30 \mathrm{ml}$.

Enzymatic hydrolysis and fractionation. A proteolytic enzyme, actinase from Streptomyces griseus (Kaken Chem Co., Tokyo, Japan), was used for enzymatic degradation. The silk fibroin solution and 5\% actinase with respect to the weight of the fibroin were mixed under nitrogen gas at $55^{\circ} \mathrm{C}$ for $12 \mathrm{~h}$. Then, the solution was heated in a boling water bath to stop the enzyme reaction and centrifuged at 5,000 rpm for $10 \mathrm{~min}$. Recycling HPLC was performed to fractionate the enzymehydrolyzed silk fibroin on a JAI-908-C60 HPLC apparatus (Japan Analytical Industry Co., Tokyo, Japan) equipped with a JAI refractive-index and UV detectors operating at $220 \mathrm{~nm}$. Both a commercially available and prepacked PVA HP-GPC column (JAI-GEL GS-220, $100 \mathrm{~cm}$ x $5 \mathrm{~cm}$ i.d.) and an ODS-BP column (JAI-GEL, $100 \mathrm{~cm}$ x $5 \mathrm{~cm}$ i.d.) were employed. Water was used as the eluting solvent at a flow rate of $3 \mathrm{ml} / \mathrm{min}$; the sample injection volume was $20 \mathrm{ml}$. The hydrolysate silk fibroin was concentrated and stored at $-20^{\circ} \mathrm{C}$ until use.

Cell culture and induction of osteoclast differentiation. Mouse monocyte/macrophage RAW 264.7 cells were purchased from the American Type Culture Collection (ATCC, Manassas, VA, USA) and maintained in DMEM supplemented with $10 \%$ FBS and $1 \%$ antibiotics in a humidified atmosphere of $5 \% \mathrm{CO}_{2}$ at $37^{\circ} \mathrm{C}$, with a media change every three days. In order to induce differentiation into osteoclasts, RAW 264.7 cells were resuspended in DMEM supplemented with $10 \% \mathrm{FBS}$ and $50 \mathrm{ng} / \mathrm{ml}$ RANKL (Petrotech Inc., Seoul, Korea) and plated in a 24-well plate. Multinucleated osteoclasts were observed starting on differentiation Day 3.

Cell viability assay. RAW 264.7 cells were treated with varying doses $(0.001,0.01,0.1,1$ and $10 \mathrm{mg} / \mathrm{ml})$ of hydrolysate silk fibroin for 24,48 and $72 \mathrm{~h}$. After treatment, the cells were incubated with the MTT solution for $3 \mathrm{~h}$ at $37^{\circ} \mathrm{C}$. The supernatants were carefully aspirated, $200 \mu \mathrm{l}$ DMSO was added to each well, and the plates were agitated to dissolve the crystal product. Absorbance was measured at $570 \mathrm{~nm}$ using the VersaMax multi-well plate reader (Molecular Devices Corp., Sunnyvale, CA, USA).

Western blot analysis. RAW 264.7 cells were washed twice with ice-cold PBS and lysed with extraction buffer containing $20 \mathrm{mM}$ Tris, $\mathrm{pH} 8,150 \mathrm{mM} \mathrm{NaCl}, 10 \mathrm{mM}$ sodium phosphate, $100 \mu \mathrm{M}$ sodium vanadate, $100 \mu \mathrm{M}$ ammonium molybdate, $10 \%$ glycerol, $0.1 \%$ Nonidet P-40, $0.1 \%$ SDS, and $1 \mathrm{X}$ protease and phosphatase inhibitors. Insoluble materials were removed by 


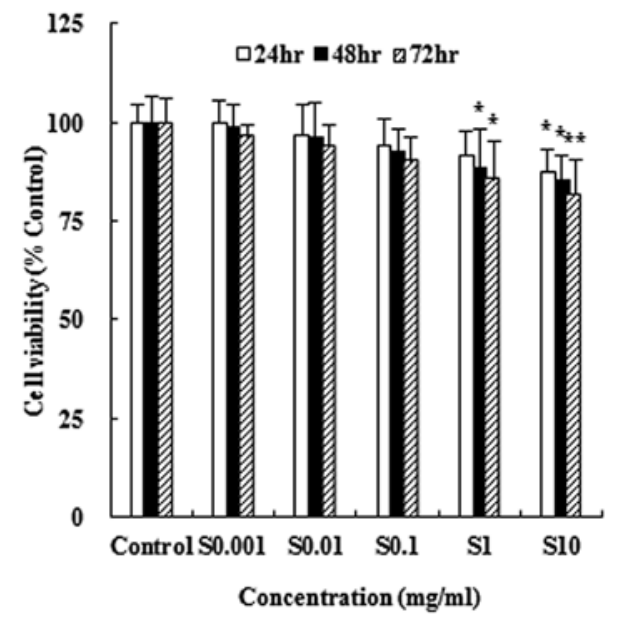

Figure 1. Effect of hydrolysate silk fibroin on RAW 264.7 cell viability. Cells were incubated with hydrolysate silk fibroin at the indicated concentrations $(0.001,0.01,0.1,1$ and $10 \mathrm{mg} / \mathrm{ml})$ for 24,48 and $72 \mathrm{~h}$ and viability was assessed by the MTT assay. Data are expressed as growth rate of cells cultured in the presence of hydrolysate silk fibroin compared to the percentage of that for untreated control cells, taken as $100 \%$. All values are mean \pm SD Values with different superscripts are significantly different by ANOVA with the Duncan's multiple range test at $\mathrm{P}<0.05$.

centrifugation at $12,000 \mathrm{rpm}$ for $20 \mathrm{~min}$. The total concentration of extracted proteins was determined using the Bradford method (28). The proteins in the supernatants $(25 \mu \mathrm{g} / \mathrm{lane})$ were electrophoretically separated on SDS-polyacrylamide gels (10-12\% SDS-PAGE) and transferred to nitrocellulose membranes. Blots were blocked in 5\% skim milk diluted in PBS-T buffer (PBS containing 0.5\% Tween-20) for $1 \mathrm{~h}$ and then incubated overnight with polyclonal antibodies against TRAF6, Erk1/2, C-Fos, NFATc1, IкB $\alpha$, NF-кB, MMP-9, CTR, cathepsin-K, poly ADP-ribose polymerase (PARP), cytochrome c, Bcl-2, Bax, caspase-3, and $\beta$-actin (Cell Signaling Technology, Beverly, MA, USA). To detect the antigen-bound antibody, the blots were treated with secondary antibody conjugated with horseradish peroxidase (HRP) coupled anti-IgG. Immunoreactive proteins were visualized by an enhanced chemiluminescence (ECL) detection system, and band intensity was quantified by densitometry (Biomaging systems ver.4.8, UVP Inc., San Gabriel, CA, USA).

Annexin V/propidium iodide (PI) flow cytometric analysis. Phosphatidylserine exposed on the outside of apoptotic cells was determined by an Annexin V-fluorescein isothiocyanate (FITC) apoptosis kit (Roche Molecular Diagnostics, Mannheim, Germany). To detect apoptosis and necrosis induced by silk fibroin in RAW 264.7 cells, the cells were treated with different concentrations of hydrolysate silk fibroin $(0.001,0.01,0.1,1$ and $10 \mathrm{mg} / \mathrm{ml})$ for $72 \mathrm{~h}$. After treatment the cells were harvested, washed twice with ice-cold PBS, and centrifuged. The cells were then stained with $100 \mu \mathrm{l}$ Annexin V-FITC labeling solution supplemented with $20 \mu \mathrm{l}$ Annexin V-FITC labeling reagent, $20 \mu 1 \mathrm{PI}$ solution, and $1 \mathrm{ml}$ incubation buffer at room temperature for $15 \mathrm{~min}$ in the dark. Annexin V-FITC and PI emissions were detected in the FL1 and FL2 channels of a FACScan flow cytometer (BD Biosciences, San Jose, CA, USA) using emission filters of 525
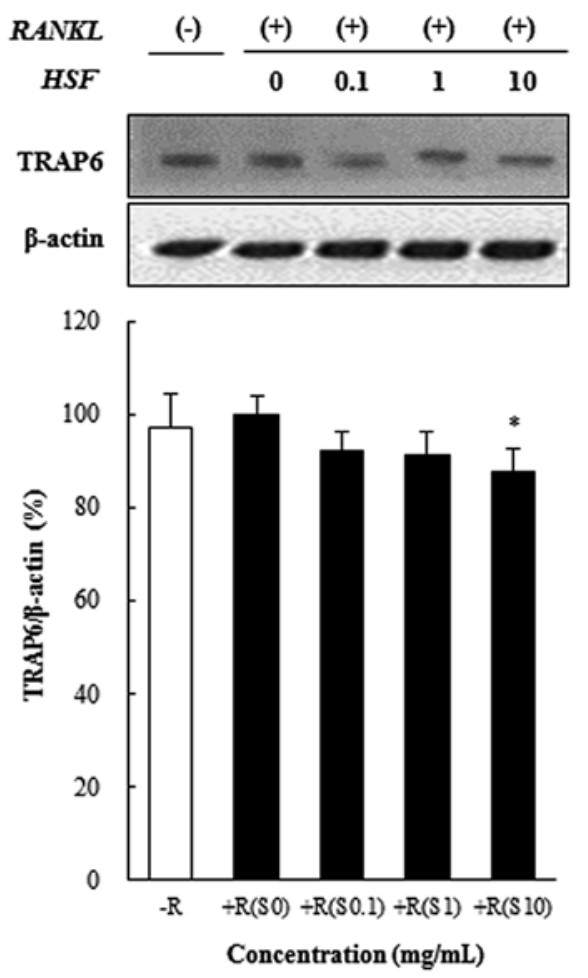

Figure 2. The effect of hydrolysate silk fibroin on osteoclastogenesis. RAW 264.7 cells were cultured with the indicated concentration of hydrolysate silk fibroin (HSF) in the presence of RANKL (50 ng/ml). After 6 days, the expression of TRAF6 was determined by immunoblotting and compared with that of $\beta$-actin. The results shown are representative of three independent experiments

and $575 \mathrm{~nm}$, respectively. The percentage distribution of intact (Annexin V-FITC-PI-), early apoptotic (Annexin V-FITC ${ }^{+} /$ $\left.\mathrm{PI}^{-}\right)$, late apoptotic (Annexin V-FITC ${ }^{+} / \mathrm{PI}^{+}$), and necrotic cells (Annexin V-FITC-/PI ${ }^{+}$) was calculated using the CellQuest ${ }^{\mathrm{TM}}$ software (BD Biosciences).

Statistical analysis. Each experiment was performed in triplicate and the results are expressed as the mean $\pm \mathrm{SD}$. Statistical analysis was performed using the SPSS software. Analysis of variance was performed using ANOVA procedures. Significant differences $(\mathrm{P}<0.05)$ between the means were determined by the Duncan's multiple range tests.

\section{Results}

Effect of hydrolysate silk fibroin on RAW 264.7 cell viability. In order to measure the cytotoxic effect of hydrolysate silk fibroin on the murine monocyte/macrophage cell line RAW 264.7, we treated cells with different concentrations of hydrolysate silk fibroin for 24, 48 and $72 \mathrm{~h}$ and measured cell viability using the MTT assay. As shown in Fig. 1, hydrolysate silk fibroin significantly reduced the viability of RAW 264.7 cells in a dose- and time-dependent manner. However, the extent of inhibition did not exceed 50\%.

Hydrolysate silk fibroin inhibits osteoclast differentiation in RAW 264.7 cells. To investigate the effect of hydrolysate silk fibroin on osteoclastogenesis of RAW 264.7 cells, cells were grown in the presence of RANKL $(50 \mathrm{ng} / \mathrm{ml})$ to induce 

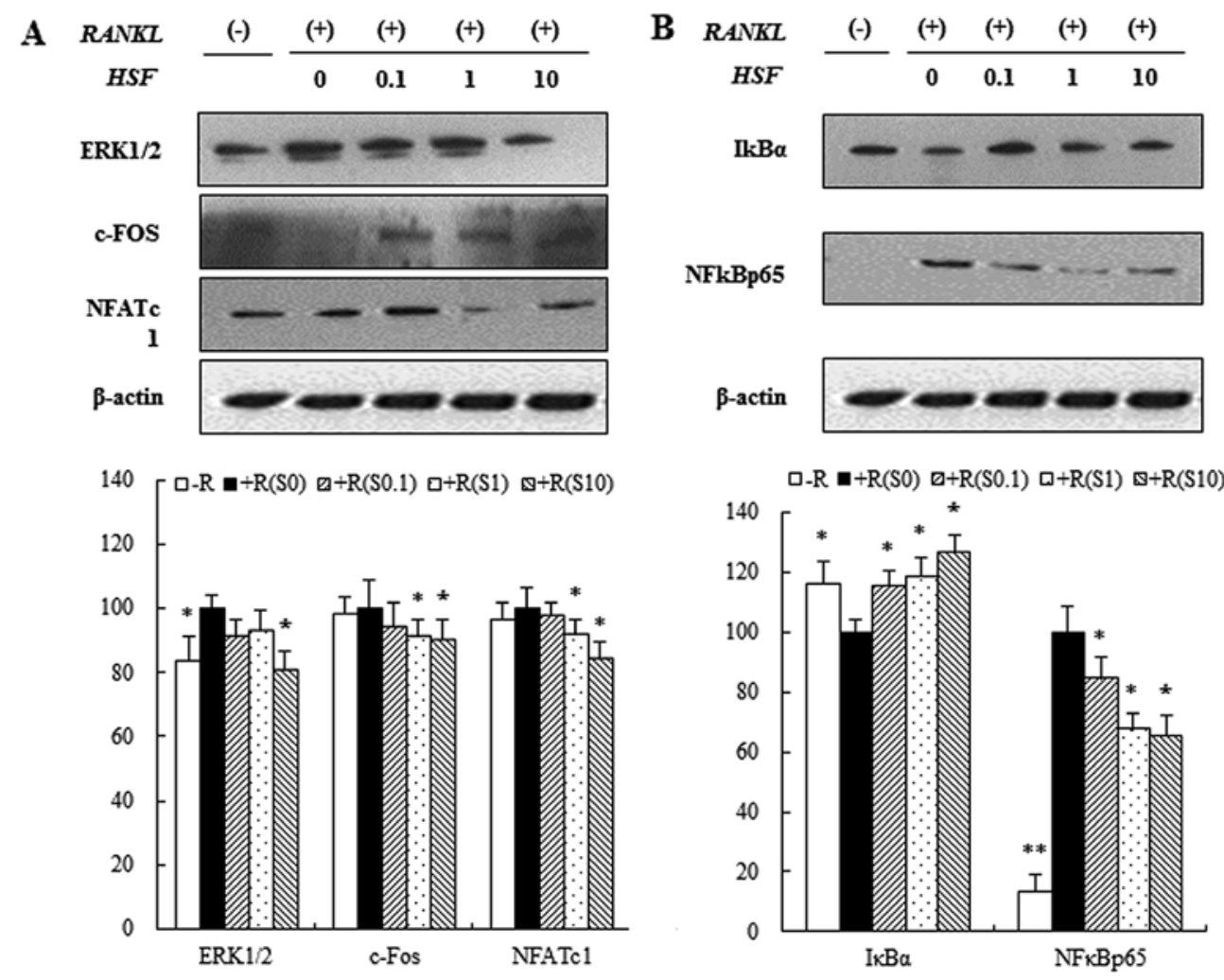

Figure 3. Effects of hydrolysate silk fibroin on activation of (A) the ERK signaling pathway, and (B) the NF-kBp65 subunit and IkB in RAW 264.7 cells. RAW 264.7 cells were treated with hydrolysate silk fibroin (HSF, $0.1,1$ and $10 \mathrm{mg} / \mathrm{ml}$ ) and were either stimulated with RANKL (+) or not (-). Cells were subjected to immunoblotting with antibodies against (A) ERK, c-Fos and NFATc1, or (B) IкB $\alpha, N K-\kappa B$, and $\beta$-actin. Relative levels of the indicated proteins were quantified by densitometric analysis and normalized to $\beta$-actin. The results shown are representative of three independent experiments.

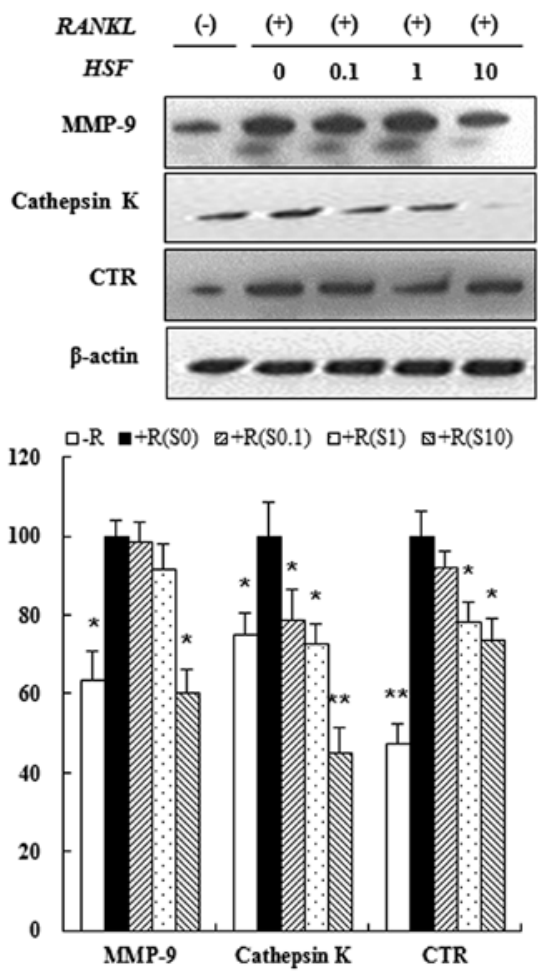

Figure 4. Effects of hydrolysate silk fibroin on the expression of osteoclastic marker proteins in RAW 264.7 cells stimulated with RANKL. RAW 264.7 cells were stimulated with RANKL (+) or not (-), treated with hydrolysate silk fibroin (HSF, $0.1,1$ and $10 \mathrm{mg} / \mathrm{ml}$ ), and analyzed by immunoblotting with antibodies against MMP-9, cathepsin-K, calcitonin receptor (CTR), and $\beta$-actin. The results shown are representative of three independent experiments. the activation of TRAF6 through the binding of RANKL to RANK. Activated TRAF6 is known to induce the expression of c-Fos, NFATc1, and NF- $\mathrm{kB}$, which are essential for osteoclastogenesis. Hydrolysate silk fibroin significantly inhibited the RANKL-induced activation of TRAF6 in preosteoclasts, as shown in Fig. 2.

Effects of hydrolysate silk fibroin on the ERK signaling pathway, NF- $\kappa B p 65$ subunit, and I $\kappa B$ in RAW 264.7 cells. Three mitogen-activated protein kinases (p38, ERK and JNK) have been implicated in the mediation of RANKL-regulated osteoclastogenesis (29). To investigate the signaling pathways by which hydrolysate silk fibroin inhibits osteoclastogenesis, we evaluated the effect of hydrolysate silk fibroin on the signaling pathways of ERK and NF- $\kappa B$. Through the ERK1/2 signaling pathway, RANKL $(50 \mathrm{ng} / \mathrm{ml})$ induced the expression of ERK1/2 in RAW 264.7 cells. This expression of ERK1/2 was attenuated by hydrolysate silk fibroin. Since activated ERK1/2 induces expression of c-Fos and NFATc1 proteins, which are essential for osteoclastogenesis, we examined the effects of hydrolysate silk fibroin on the expression of c-Fos and NFATc1. RANKL induced the expression of c-Fos and NFATc1, but treatment with hydrolysate silk fibroin significantly inhibited RANKL-induced c-Fos and NFATc1 expression (Fig. 3A). Activation of NF- $\mathrm{kB}$ is also involved in osteoclastogenesis (30). NF- $\kappa \mathrm{B}$, a key transcription factor for osteoclastogenesis, is activated via the IKB kinase signaling pathway (12) and translocation of the NF- $\mathrm{kBp} 65$ subunit into the nucleus is induced by RANKL. Our data showed that 
A
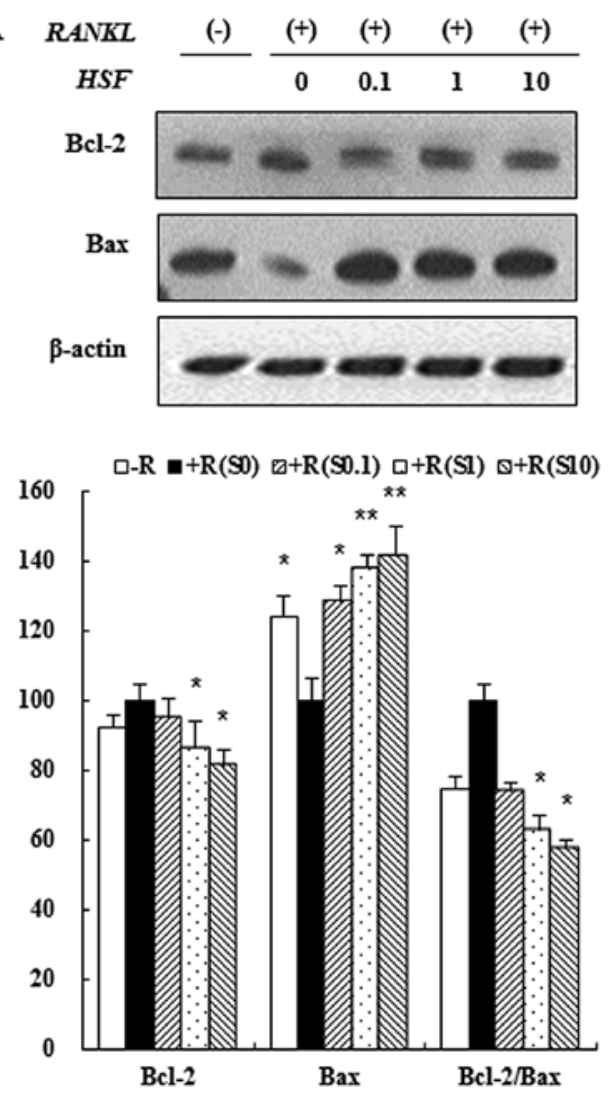
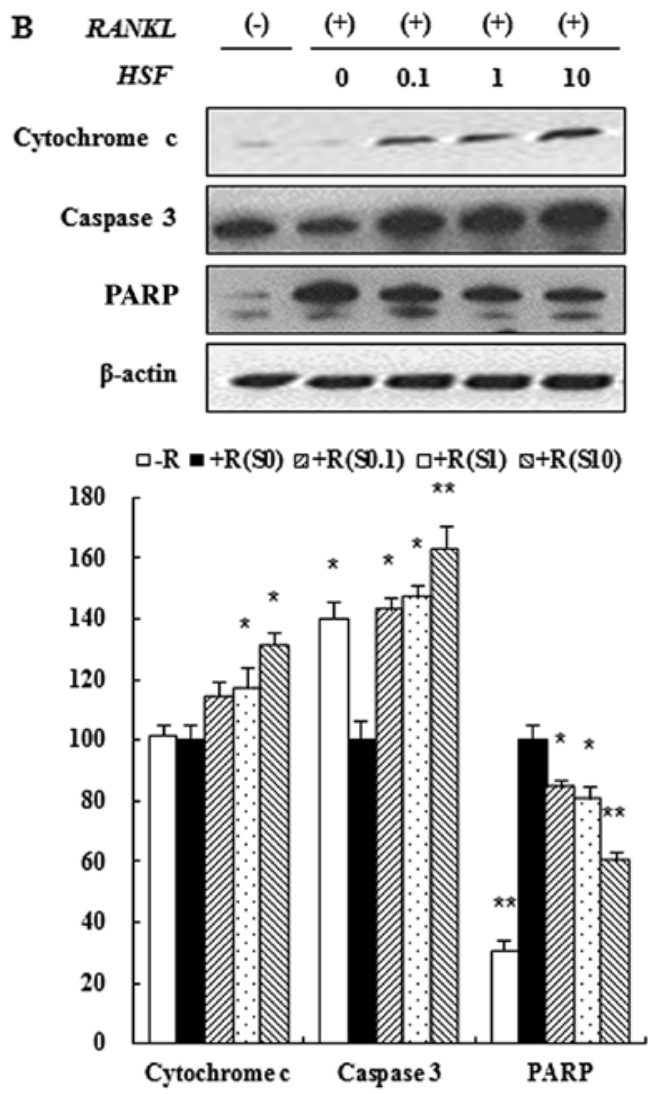

Figure 5. Effect of hydrolysate silk fibroin on activation/expression of apoptotic signaling molecules in osteoclasts. RAW 264.7 cells were treated with hydrolysate silk fibroin (HAF, 0.1, 1 and $10 \mathrm{mg} / \mathrm{ml}$ ) in the presence (+) or absence (-) of RANKL, and then analyzed by immunoblotting with antibodies against (A) $\mathrm{Bcl}-2$ and Bax, or (B) cytochrome $\mathrm{c}$, caspase-3 and PARP and $\beta$-actin. Relative levels of the indicated proteins were quantified by densitometric analysis and normalized to $\beta$-actin. The results shown are representative of three independent experiments.

hydrolysate silk fibroin inhibited NF-kBp65 expression and degradation of IkB $\alpha$ in a dose-dependent manner (Fig. 3B).

Hydrolysate silk fibroin inhibited expression of osteoclastspecific proteins. Osteoclastogenesis is associated with upregulation of specific proteins in response to RANKL (31). Therefore, we assessed the inhibitory effect of hydrolysate silk fibroin on osteoclastogenesis by evaluating the expression of osteoclast-related proteins such as MMP-9, cathepsin-K and CTR. Immunoblot analysis showed that the expression of osteoclastic protein markers was increased by RANKL, but treatment with hydrolysate silk fibroin inhibited expression of these proteins in a dose-dependent manner (Fig. 4). These results indicate that hydrolysate silk fibroin has a specific effect on the regulation of proteins that are induced during osteoclastogenesis.

Hydrolysate silk fibroin induced apoptosis of osteoclasts. Apoptosis is characterized by activation of a caspase cascade that can be induced by the release of cytochrome c. We evaluated the effect of hydrolysate silk fibroin on caspase-3 activation and cytochrome $\mathrm{c}$ release in osteoclasts. RAW 264.7 cells were incubated in the absence or presence of RANKL with hydrolysate silk fibroin $(0.1,1$ and $10 \mathrm{mg} / \mathrm{ml})$ for $72 \mathrm{~h}$. Proteins were analyzed by immunoblotting and quantified by densitometric analysis. Caspase- 3 activity was induced by hydrolysate silk fibroin at high concentrations $(10 \mathrm{mg} / \mathrm{ml})$
(Fig. 5B). Additionally, the release of cytochrome c into the cytoplasm was increased by hydrolysate silk fibroin in a dosedependent manner (Fig. 5B). Hydrolysate silk fibroin induced the cleavage/activation of caspase-3 and PARP, and decreased the expression of anti-apoptotic proteins such as Bcl-2 in a dose-dependent manner. In contrast, expression of Bax was increased by Hydrolysate silk fibroin in a dose-dependent manner (Fig. 5A).

Hydrolysate silk fibroin induces apoptosis in RAW 264.7 cells by Annexin V/PI analysis. To ascertain the mechanism of cell death (apoptosis or necrosis), RAW 264.7 cells were treated with different concentrations of hydrolysate silk fibroin $(0.001,0.01,0.1,1$ and $10 \mathrm{mg} / \mathrm{ml})$ for $72 \mathrm{~h}$, and then stained with Annexin V-FITC and PI. This protocol is based on the observation that, soon after initiating apoptosis, membrane phosphatidylserine (PS) translocates from the inner face of the plasma membrane to the cell surface where it can be easily detected by staining with a fluorescent conjugate of Annexin $\mathrm{V}$, a protein that has a very strong affinity for PS. Simultaneous staining with Annexin V-FITC and PI non-vital dye makes it possible to distinguish between intact, early apoptotic, late apoptotic, and necrotic cells. The results indicated that hydrolysate silk fibroin decreased the percentage of live cells and increased the number of early apoptotic cells. When the concentrations increased, the percentage of normal cells decreased from $65.01 \%$ (control) 


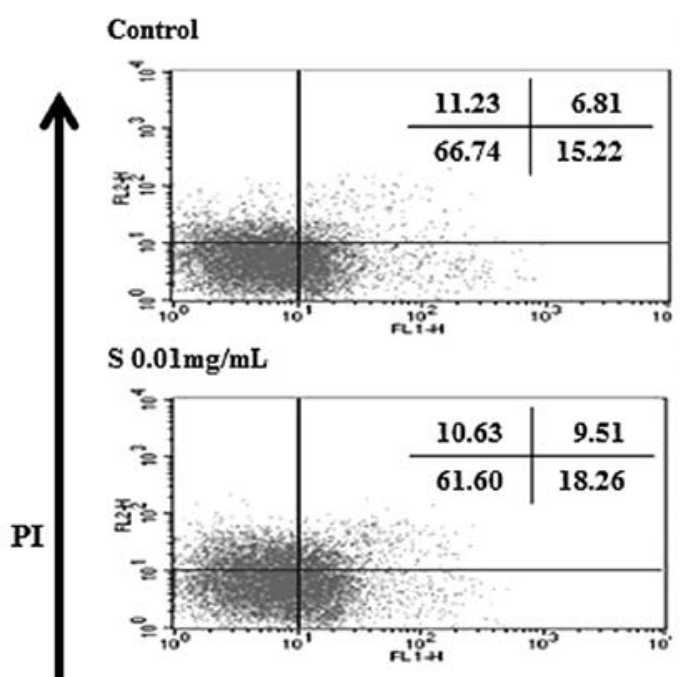

$\mathrm{S} 0.001 \mathrm{mg} / \mathrm{mL}$

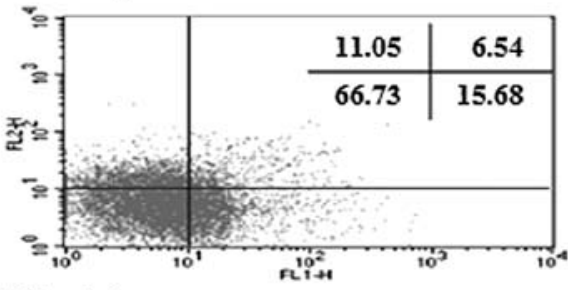

$100 \mathrm{mg} / \mathrm{mL}$

$250 \mathrm{mg} / \mathrm{mL}$

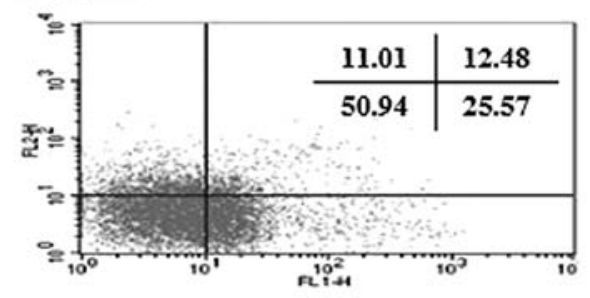

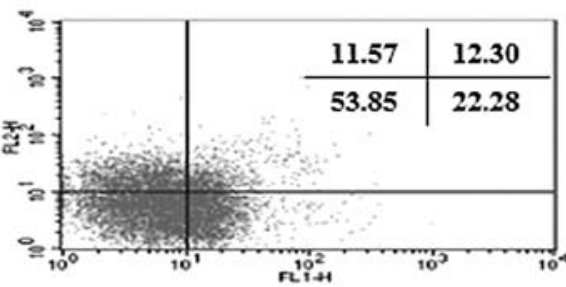

$500 \mathrm{~kg} / \mathrm{mL}$

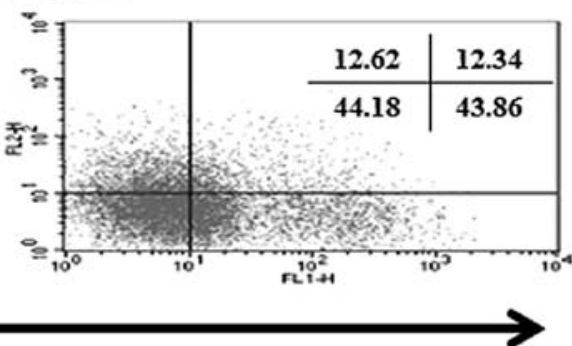

Annexin V

Figure 6. Flow cytometric analysis of Annexin V and PI double staining. Cells were incubated with hydrolysate silk fibroin at the indicated concentration $(0.001,0.01,0.1,1$ and $10 \mathrm{mg} / \mathrm{ml})$ for $72 \mathrm{~h}$ and the increase in apoptotic cells was evaluated by Annexin V/PI double staining. The percentages of apoptotic cells are indicated.

to $48.75 \%(10 \mathrm{mg} / \mathrm{ml})$. However the percentage of apoptotic cells increased from $25.17 \%$ (control) to $39.96 \%(10 \mathrm{mg} / \mathrm{ml})$ (Fig. 6). These results indicate that the decrease in live cells is due at least in part to the effect of hydrolysate silk fibroin on inducing apoptosis.

\section{Discussion}

There is growing interest about the use of natural extracts to prevent loss of bone mass by inhibiting osteoclastogenesis and bone resorptive activity, and/or inducing osteoclast apoptosis. Silk fibroin possesses several biological and pharmacological activities including reducing cholesterol, hypoglycemia, and growth of osteoblasts, and enhancing bone formation (32). A previous study reported that silk fibroin inhibits osteoclastogenesis. This study further demonstrated that osteoclastogenesis is indirectly inhibited by osteoblasts/stromal cells; however, there are no studies showing direct inhibition of osteoclastogenesis.

Therefore, the aims of this study were to evaluate the effects of hydrolysate silk fibroin on osteoclastogenesis and the induction of apoptosis. We found that hydrolysate silk fibroin is a potent inhibitor of osteoclasts in RANKL-stimulated RAW 264.7 cells. As shown in Fig. 3, the inhibitory effect of hydrolysate silk fibroin was demonstrated by measuring the RANKL-induced expression of osteoclast-related proteins such as MMP-9, cathepsin-K, and CTR, which are required for the bone resorptive activity of mature osteoclasts. MMP-9 and cathepsin-K are highly expressed in osteoclasts. MMP-9 can initiate the osteoclastic resorption process by removing the collagenous layer from the bone surface (33), while cathepsin-K plays an essential role in osteoclastic bone resorption $(34,35)$. Therefore, these results suggest that hydrolysate silk fibroin could inhibit the resorbing capacity of osteoclasts.

The effects of hydrolysate silk fibroin on the signaling mechanism of RANKL were clearly demonstrated. The inhibitory effect of hydrolysate silk fibroin on the differentiation stage results from suppression of RANKL-induced ERK1/2 signaling and expression of NF- $\mathrm{Bp} 65$. Binding of RANKL to RANK stimulates TRAF6 and various transcription factors (36). TRAF6 activation subsequently leads to the activation of MAP kinases (ERK, JNK and p38) and NF- $\kappa \mathrm{B}$, which induces the expression of proteins required for osteoclastogenesis (37). Several transcription factors have been suggested to regulate induction of genes involved in RANKL-induced osteoclastogenesis (38). Importantly, RANKL specifically and strongly induces NFATc1, the master regulator of osteoclastogenesis, and this induction is dependent on c-Fos pathways (39). RANKL has been shown to elevate the level of c-Fos expression in osteoclasts, and our data indicated that hydrolysate silk fibroin inhibits this RANKL-induced c-Fos expression (Fig. 4A). We therefore investigated whether hydrolysate silk fibroin reduced the activation of NFATc1 in osteoclastogenesis in RAW 264.7 cells. As shown in Fig. 4A, hydrolysate silk fibroin reduced the transcriptional activity of NFATc1. These results suggest that hydrolysate silk fibroin suppressed 
NTATc1 by inhibiting the upregulation of c-Fos in response to RANKL. When RANKL-stimulated RAW 264.7 cells were treated with hydrolysate silk fibroin, MMP-9 expression was inhibited in a response similar to that of NFATc1 expression. NFATc1 was proposed to act as a downstream mediator of increased MMP-9 expression during osteoclastogenesis (40). Therefore, our results suggest that the inhibitory effect of hydrolysate silk fibroin on MMP-9 may be responsible for the downregulation of NFATc1 activation.

As mentioned above, NF- $\kappa \mathrm{B}$ is another transcription factor that is activated by the RANKL signaling pathway in osteoclastogenesis. In the absence of RANKL, NF- $\kappa \mathrm{B}$ is retained in the cytoplasm as a complex with the I $\mathrm{B}$ protein. However, when the cells are stimulated by RANKL, the I $\kappa B$ kinase (IKK) complex phosphorylates I $\mathrm{B}$ and the phosphorylated $\mathrm{I} \kappa \mathrm{B}$ is targeted by the proteasome and subsequently degraded, leading to the activation of $\mathrm{NF}-\kappa \mathrm{B}$ signaling. The $\mathrm{NF}-\kappa \mathrm{Bp} 65$ subunit is separated from $\mathrm{I} \kappa \mathrm{B} \alpha$ and enters the nucleus where it enhances transcription of target genes (41). Therefore, inhibition of $\mathrm{NF}-\kappa \mathrm{B}$ translocation might be an effective approach to target osteoclastogenesis. In our study, hydrolysate silk fibroin inhibited RANKL-induced I $\mathrm{B}$ degradation and activation of NF- $\kappa$ Bp65 in the cytoplasm. These findings raise the possibility that silk fibroin prevents the translocation of the NF- $\kappa$ Bp65 subunit through the blockade of phosphorylationinduced I $\kappa \mathrm{B} \alpha$ degradation.

In addition to its effects on osteoclastogenesis, hydrolysate silk fibroin also induced apoptosis in osteoclasts. Osteoclasts are characterized by multinuclearity, an actin ring structure, and acidic cell conditions during resorption. Osteoclasts can be inhibited by disrupting the actin ring, triggering their apoptosis, and/or inhibiting expression/activity of proteins required for their function (42). The classical Fas signaling pathway involving mitochondrial release of cytochrome $\mathrm{c}$ and activation of caspase- 3 has been identified in mouse and human osteoclasts and in RAW 264.7 cells (9). Therefore, we investigated the effect of hydrolysate silk fibroin on the activation/ expression of apoptotic signaling molecules in osteoclasts by immunoblot analysis of RAW 264.7 cells. As shown in Fig. 5, the Fas signaling pathway was induced by hydrolysate silk fibroin. In addition, anti-apoptotic Bcl-2 was downregulated and pro-apoptotic Bax was upregulated by treatment with hydrolysate silk fibroin. These results suggest that hydrolysate silk fibroin has antiresorptive activity by both inhibiting osteoclastogenesis and inducing apoptosis of osteoclasts. Although our findings are preliminary and have certain limitations, our study may provide new insight toward understanding the mechanisms of osteoclastogenesis and apoptosis.

In conclusion, we suggest that hydrolysate silk fibroin modulates expression of specific genes in osteoclasts through downregulation of transcription factors such as NFATc1 and $\mathrm{NF}-\kappa \mathrm{B}$, and also induces apoptosis of osteoclasts. Therefore, hydrolysate silk fibroin may be a useful natural compound for reducing osteoclastogenesis and preventing bone resorption.

\section{Acknowledgements}

This study was supported by 2012 Post-doctoral Fellowship Program (PJ906973022012) of National Academy Science, Rural Development Administration, Republic of Korea.

\section{References}

1. Parfitt AM: Bone remodeling and bone loss: Understanding the pathophysiology of osteoporosis. Clin Obstet Gynecol 30: 789-811, 1987.

2. Boyle WJ, Simonet WS and Lacey DL: Osteoclast differentiation and activation. Nature 423: 337-342, 2003.

3. Matsuo K and Irie N: Osteoclast-osteoblast communication. Arch Biochem Biophys 473: 201-209, 2008.

4. Darnay BG and Aggarwal BB: Signal transduction by tumour necrosis factor and tumour necrosis factor related ligands and their receptors. Ann Rheum Dis 58: 12-113, 1999.

5. Koga T, Inui M, Inoue K, Kim SH, Suematsu A, Kobayashi E, Iwata T, Ohnishi H, Matozaki T, Kodama T, et al: Costimulatory signals mediated by the ITAM motif cooperate with RANKL for bone homeostasis. Nature 428: 758-763, 2004.

6. Anusaksathien O, Laplace C, Li X, Ren Y, Peng L, Goldring SR and Galson DL: Tissue-specific and ubiquitous promoters direct the expression of alternatively spliced transcripts from the calcitonin receptor gene. J Biol Chem 276: 22663-22674, 2001.

7. Boyce BF, Wright K, Reddy SV, Koop BA, Story B, Devlin R, Leach RJ, Roodman GD and Windle JJ: Targeting simian virus $40 \mathrm{~T}$ antigen to the osteoclast in transgenic mice causes osteoclast tumors and transformation and apoptosis of osteoclasts. Endocrinology 136: 5751-5759, 1995.

8. Hughes DE and Boyce BF: Apoptosis in bone physiology and disease. Mol Pathol 50: 132-137, 1997.

9. Wu X, McKenna MA, Feng X, Nagy TR and McDonald JM: Osteoclast apoptosis: the role of fas in vivo and in vitro. Endocrinology 144: 55445-5555, 2003.

10. Xing L and Boyce BF: Regulation of apoptosis in osteoclasts and osteoblastic cells. Biochem Biophys Res Commun 328: 709-720, 2005.

11. Rodan GA amd Martin TJ: Therapeutic approaches to bone diseases. Science 289: 1508-1514, 2000.

12. Yamashita T, Yao Z, Li F, Zhang Q, Badell IR, Schwarz EM, Takeshita S, Wagner EF, Noda M and Matsuo K: NF-kappaB p50 and p52 regulate receptor activator of NF-kappaB ligand (RANKL) and tumor necrosis factor-induced osteoclast precursor differentiation by activating c-fos and NFATc1. J Biol Chem 282: 18245-18253, 2007.

13. Park KH, Ju WC and Cho YH: Conditioned medium of soybean extract treated osteoblasts inhibits RANKL induced differentiation of osteoclasts. FASEB J 39: 64-70, 2010.

14. Shin JM, Park CK, Shin EJ, Cho TH and Hwang IK: Effects of Scutellaria radix extract on osteoblast differentiation and osteoclast formation. Korean J Food Sci Tech 40: 674-679, 2008.

15. Wang G, Yang H, Li M, Lu S, Chen X and Cai X: The use of silk fibroin/hydroxyapatite composite co-cultured with rabbit bonemarrow stromal cells in the healing of a segmental bone defect. J Bone Joint Surg Br 92: 320-325, 2010.

16. Zhou C, Confalonieri F, Jacquet M, Perasso R, Li Z and Janin J: Silk fibroin: structural implications of a remarkable amino acid sequence. Proteins 44: 119-122, 2001.

17. Kim ED, Bayaraa T, Shin EJ and Hyun CK: Fibroin-derived peptides stimulate glucose transport in normal and insulinresistant 3T3-L1 adipocytes. Biol Pharm Bull 32: 427-433, 2009.

18. Tsukada M, Freddi G, Minoura N and Allara G: Preparation and application of porous silk fibroin materials. J Appl Polym Sci 54: 507-514, 1994.

19. Nishida A, Yamada M, Kanazawa T, Takashima Y, Ouchi K and Okada H: Use of silk protein, sericin, as a sustained-release material in the form of a gel, sponge and film. Chem Pharm Bull 58: 1480-1486, 2010.

20. Kato N, Sato S, Yamanaka A, Yamada H, Fuwa N and Nomura M: Silk protein, sericin, inhibits lipid peroxidation and tyrosinase activity. Biosci Biotechnol Biochem 62: 145-147, 1998.

21. Acharya C, Ghosh SK and Kundu SC: Silk fibroin protein from mulberry and non-mulberry silkworms: cytotoxicity, biocompatibility and kinetics of L929 murine fibroblast adhesion. J Mater Sci Mater Med 19: 2827-2836, 2008.

22. Minoura N, Aiba S, Gotoh Y, Tsukada M and Imai Y: Attachment and growth of cultured fibroblast cells on silk protein matrices. $\mathbf{J}$ Biomed Mater Res 29: 1215-1221, 1995.

23. Sofia S, McCarthy MB, Gronowiza G and Kaplan DL: Functionalized silk-based biomaterials for bone formation. J Biomed Mater Res 54: 139-148, 2001. 
24. Roh DH, Kang SY, Kim JY, Kwon YB, Young Kweon H, Lee KG, Park YH, Baek RM, Heo CY, Choe J, et al: Wound healing effect of silk fibroin/alginate-blended sponge in full thickness skin defect of rat. J Biomed Mater Res 17: 547-552, 2006.

25. Hanawa T, Watanabe A, Tsuchiya T, Ikoma R, Hidaka M and Sugihara M: New oral dosage form for elderly patients. II. Release behavior of benfotiamine from silk fibroin gel. Chem Pharm Bull (Tokyo) 43: 872-876, 1995.

26. Griffith LG and Naughton G: Tissue engineering - current challenges and expanding opportunities. Science 295: 1009-1014, 2002.

27. Yeo JH, Park KH, Ju WC, Lee JA, Lee GG, Woo SO, Han SM, Kweon HY, Kim SS and Cho YH: Inhibitory effect of conditioned medium of silk fibroin-treated osteoblasts in osteoclast differentiation. J Korean Soc Food Sci Nutr 37: 992-997, 2008.

28. Bradford MM: A rapid and sensitive method for the quantitation of microgram quantities of protein utilizing the principle of protein-dye binding. Anal Biochem 72: 248-254, 1976.

29. Matsuzaki K, Udagawa N, Takahashi N, Yamaguchi K, Yasuda H, Shima N, Morinaga T, Toyama Y, Yabe Y, Higashio K, et al: Osteoclast differentiation factor (ODF) induces osteoclastlike cell formation in human peripheral blood mononuclear cell cultures. Biochem Biophys Res Commun 246: 199-204, 1998.

30. Jimi E, Akiyama S, Tsurukai T, Okahashi N, Kobayashi K, Udagawa N, Nishihara T, Takahashi N and Suda T: Osteoclast differentiation factor acts as a multifunctional regulator in murine osteoclast differentiation and function. J Immunol 163: 434-442, 1999.

31. Lee SE, Woo KM, Kim SY, Kim H, Kwack K, Lee ZH and Kim H: The phosphatidylinositol 3-kinase, p38, and extracellular signal-regulated kinase pathways are involved in osteoclast differentiation. Bone 30: 71-77, 2002.

32. Altman GH, Diaz F, Jakuba C, Calabro T, Horan RL, Chen J, Lu H, Richmond J and Kaplan DL: Silk-based biomaterials. Biomaterials 24: 401-416, 2003.
33. Reponen P, Sahlberg C, Munaut C, Thesleff I and Tryggvason K: High expression of $92-\mathrm{kD}$ type IV collagenase (gelatinase B) in the osteoclast lineage during mouse development. J Cell Biol 124: 1091-1102, 1994.

34. Gelb BD, Shi GP, Chapman HA and Desnick RJ: Pycnodysostosis, a lysosomal disease caused by cathepsin K deficiency. Science 279: 1236-1238, 1996.

35. Ishikawa T, Kamiyama M, Tani-Ishii N, Suzuki H, Ichikawa Y, Hamaguchi Y, Momiyama $\mathrm{N}$ and Shimada $\mathrm{H}$ : Inhibition of osteoclast differentiation and bone resorption by cathepsin $\mathrm{K}$ antisense oligonucleotides. Mol Carcinog 32: 84-91, 2001.

36. Kanazawa K and Kudo A: TRAF2 is essential for TNF-alphainduced osteoclastogenesis. J Bone Miner Res 20: 840-847, 2005.

37. Lee ZH and Kim H: Signal transduction by receptor activator of nuclear factor kappa B in osteoclasts. Biochem Biophys Res Commun 305: 211-214, 2003.

38. Takayanagi H, Kim S, Koga T, Nishina H, Isshiki M, Yoshida H, Saiura $\mathrm{A}$, Isobe $\mathrm{M}$, Yokochi $\mathrm{T}$, Inoue $\mathrm{J}$, et al: Induction and activation of the transcription factor NFATc1 (NFAT2) integrate RANKL signaling in terminal differentiation of osteoclasts. Dev Cell 3: 889-901, 2002.

39. Karsenty G and Wagner EF: Reaching a genetic and molecular understanding of skeletal development. Dev Cell 2: 389-406, 2002.

40. Ishida N, Hayashi K, Hoshijima M, Ogawa T, Koga S, Miyatake Y, Kumegawa M, Kimura T and Takeya T: Large scale gene expression analysis of osteoclastogenesis in vitro and elucidation of NFAT2 as a key regulator. J Biol Chem 277: 41147-41156, 2002.

41. Matsuo K, Galson DL, Zhao C, Peng L, Laplace C, Wang KZ, Bachler MA, Amano H, Aburatani H and Ishikawa H: Nuclear factor of activated T-cells (NFAT) rescues osteoclastogenesis in precursors lacking c-fos. J Biol Chem 279: 26475-26480, 2004.

42. Stern PH: Antiresorptive agents and osteoclast apoptosis. J Cell Biochem 101: 1087-1096, 2007. 